\title{
Percepción sobre la vida silvestre desde diversas visiones religiosas: Una herramienta para promover la educación ambiental en la Península de Osa
}

\author{
Ana Clara Mazzolari, Laín Pardo, Otto Monge, Víctor Montalvo, Dora Armero-Durán, Juan Carlos Cruz-Díaz, \\ Nancy Orias-Hidalgo, Virginia Rodríguez, Martha Sarmento, Adriana Tovar, Emilio Vargas-Mena y Grace Wong-Reyes \\ Instituto Internacional en Conservación y Manejo de Vida Silvestre, Universidad Nacional de Costa Rica, Apdo. 1350-3000 Heredia, Costa Rica; \\ mazzolari84@yahoo.com.ar; lepardov@yahoo.com; monge_otto@hotmail.com; vmontalvog@gmail.com; doris.armero@gmail.com; \\ pj_jccd@hotmail.com; nohidalgo@gmail.com; virginiallamas@yahoo.com.ar; marthacms@gmail.com; aetovarm@gmail.com; \\ evargasmena@gmail.com; gwongr@gmail.com
}

Recibido 17-V-2012 Corregido 10-VIII-2012 Aceptado 4-IX-2012

\begin{abstract}
Perception of wildlife from various religious views: A tool to promote environmental education in the Osa Peninsula. In the Osa peninsula in southern Costa Rica, hunting and wildlife trade are common practices that threaten biodiversity conservation. Religious leaders could play an important role if they integrate in their speeches ideas about these issues, as they tend to create deep links with the community. In order to understand the perception of religious leaders against local environmental issues they were interviewed in depth. In general they showed sensitivity to nature and the current environmental problems. They showed an acceptance on subsistence hunting, but not for commercial or sport hunting. Also religious leaders showed a willingness to learn about environmental issues and ways to mitigate them, as well as elements in their doctrines that can be used to channel environmental education strategies.
\end{abstract}

KEY WORDS

Religion, hunting, religious awareness, environmental education, wildlife, Osa Peninsula.

\section{RESUMEN}

En la península de Osa, al sur de Costa Rica, la cacería y el comercio de la fauna silvestre son prácticas extendidas que ponen en riesgo su conservación. Los líderes religiosos podrían ser actores importantes si se integra en sus discursos ideas sobre esta problemática, pues ellos tienden a crear enlaces profundos con la comunidad. Con el objetivo de conocer la percepción de los líderes religiosos frente a la problemática ambiental local, se realizaron entrevistas a profundidad. En general mostraron sensibilidad hacia la naturaleza y los problemas ambientales actuales. Existió una aceptación sobre la caza de subsistencia, pero no así de la caza deportiva ni comercial. Se encontró una buena disposición de aprender sobre la problemática ambiental y las vías para su mitigación, así como elementos en sus doctrinas que pueden usarse para canalizar estrategias de educación ambiental.

\section{PALABRAS CLAVE}

Religión, cacería, percepción religiosa, educación ambiental, vida silvestre, Península de Osa.
La cacería y el comercio de la fauna silvestre constituyen unas de las causas más importantes de la pérdida de biodiversidad en Latinoamérica (Pérez \& Ojasti, 1996). En Costa Rica, la actividad de cacería es realizada por aproximadamente el $1,3 \%$ de la población y cerca del $13 \%$ come carne de monte al menos una vez al año. Más aún, en términos generales, la gente aprueba la cacería para subsistencia, pero consideran la cacería por deporte como una actividad cruel (Drews, 2003).
La cacería en la península de Osa ha sido desarrollada principalmente por deporte y en menor medida por subsistencia $y$, aunque esta práctica ha venido disminuyendo en esta zona debido a los controles de las autoridades ambientales, todavía se sigue manteniendo por tradición familiar (Agenda XXI-ACOSA, 2004). No obstante líderes comunales y pobladores de la península han declarado que la cacería es el principal problema que afecta la conservación de la fauna en la región (Hernández et al., 2008; Hernández et al., 2011). 
En Costa Rica, se ha incentivado el turismo ecológico como un modelo de desarrollo en las zonas rurales y la península de Osa, con su riqueza en biodiversidad, no es la excepción (Driscoll et al., 2011). Dentro de ese esquema de desarrollo, la conservación de la fauna silvestre en Osa es de vital importancia, ya que es uno de los elementos que atrae a los turistas a visitar la zona, a pesar de que mucha de su biodiversidad se encuentra amenazada por sobre explotación de los recursos y el desarrollo de actividades agrícolas y ganaderas (ELAP, 2005).

Una de las funciones de la educación ambiental es promover y apoyar un diálogo universal, poniendo en relieve los principios ecológicos por encima de las fronteras ideológicas y políticas; dependiendo de la participación activa de un amplio conjunto de personas e instituciones (UNESCO, 1977). Para el caso de Costa Rica, una parte importante de la Educación Ambiental (EA) es detallada en la "Estrategia Nacional para la Educación Ambiental 20052010" del Ministerio del Ambiente y Energía y del Sistema Nacional de Áreas de Conservación, basada en su propia experiencia y en el reconocimiento de tendencias internacionales y nacionales (MINAE-SINAC, 2005). En la península de Osa, aparte de los esfuerzos aislados por educar a los pobladores sobre la contaminación y manejo de basuras, es necesario implementar programas de educación ambiental sobre la pérdida de la fauna silvestre de la zona (Hernández et al., 2011).

Para abordar el tema de la cacería ilegal de vida silvestre, es necesario conocer la opinión de diversos actores de la sociedad como pobladores, educadores, el sector económico y líderes religiosos. Estos últimos podrían ser capaces de crear enlaces más profundos con los otros actores y así, desde cada congregación, generar un espacio ideal de comunicación y reflexión sobre temas ambientales (Dudley et al., 2005). De esta manera, el objetivo de este trabajo fue conocer la percepción de los líderes religiosos frente a la problemática ambiental local y cómo ven desde su congregación la vida silvestre en general. Esto, con el fin de investigar el potencial aporte que pueden tener en la divulgación y concienciación de la problemática ambiental en la zona.

\section{METODOLOGÍA}

\section{Área de estudio}

La Península de Osa se localiza al sureste de Costa Rica y cuenta con una extensión de $1750 \mathrm{~km}^{2}$ (Christen, 1994), estando bajo alguna categoría de protección alrededor del $60 \%$ de los terrenos (Hernández et al., 2011). Su altitud máxima es de $782 \mathrm{msnm}$, tiene una topografía quebrada y su precipitación anual es de 3 800-5 800mm (Rosero-Bixby et al., 2002). La península tiene tres zonas de vida: bosque tropical húmedo, el bosque tropical muy húmedo y la transición de bosque pluvial a premontano (Tosi, 1969). Además constituye el último reducto de bosque tropical húmedo de la costa pacífica mesoamericana (Kappelle et al., 2002). Es reconocida de manera especial por su alta biodiversidad, conteniendo un tercio de las especies de árboles de Costa Rica con más de 4000 especies de plantas, 375 especies de aves ( 2 endémicas), 117 especies de anfibios y reptiles, 124 especies de mamíferos y alrededor de 8000 especies de insectos (Mansour, 1995; HerreraMacBryde et al., 1997).

La zona cuenta con varias áreas legalmente protegidas y contempladas por el Sistema Nacional de Áreas de Conservación (SINAC). Estas áreas incluyen: un Parque Nacional (Corcovado), una Reserva Forestal (Golfo Dulce), cuatro Refugios de Vida Silvestre Privados (Río Oro, Hacienda Cópano, Forestal Golfito S.A. y D.P. Hayes), cinco Refugios de Vida Silvestre Mixtos (Punta Río Claro, Preciosa Platanares, Pejeperro, Pejeperrito, Osa y Quilotro), un Refugio de Vida Silvestre Estatal (Golfito) y un territorio indígena (Guaymí Osa) (Fig. 1; Vargas-Mena, 2006; Wong, 2006).

En contraste con su alta biodiversidad, la península de Osa es una de las zonas de mayor pobreza en Costa Rica (Driscoll, 2011). Cuenta con una población aproximada de 10000 habitantes y corresponde a uno de los sitios con las más altas proporciones de hogares con pobreza extrema: el $13,1 \%$ de los hogares no poseen ingresos suficientes para satisfacer sus necesidades alimentarias básicas (MIDEPLAN, 2003). Además la región tiene altos niveles de mortalidad infantil, analfabetismo y según datos del 2001 el ingreso promedio mensual por habitante era uno de los más bajos del país (MIDEPLAN, 2003; Fundación Marviva, 2009).

\section{Entrevistas}

Se realizaron entrevistas a profundidad (Valles, 1997) a varios líderes religiosos pertenecientes a los pueblos de La Palma, Cañaza y Puerto Jiménez (Fig. 1), del 11 al 14 de marzo de 2010. Para la selección de los entrevistados se trató de cubrir la mayor cantidad y diversidad de líderes religiosos posible, teniendo en cuenta criterios como tipo de culto, edades y género. Se identificaron aquellos líderes más importantes dentro de la comunidad debido a que podían actuar como informantes clave y sus opiniones podían reflejar en cierta medida el pensamiento del grupo religioso al que pertenecían. Las entrevistas estuvieron enfocadas en comprender la visión de los líderes religiosos frente a la cacería y la posible incorporación de componentes de educación ambiental dentro de sus 


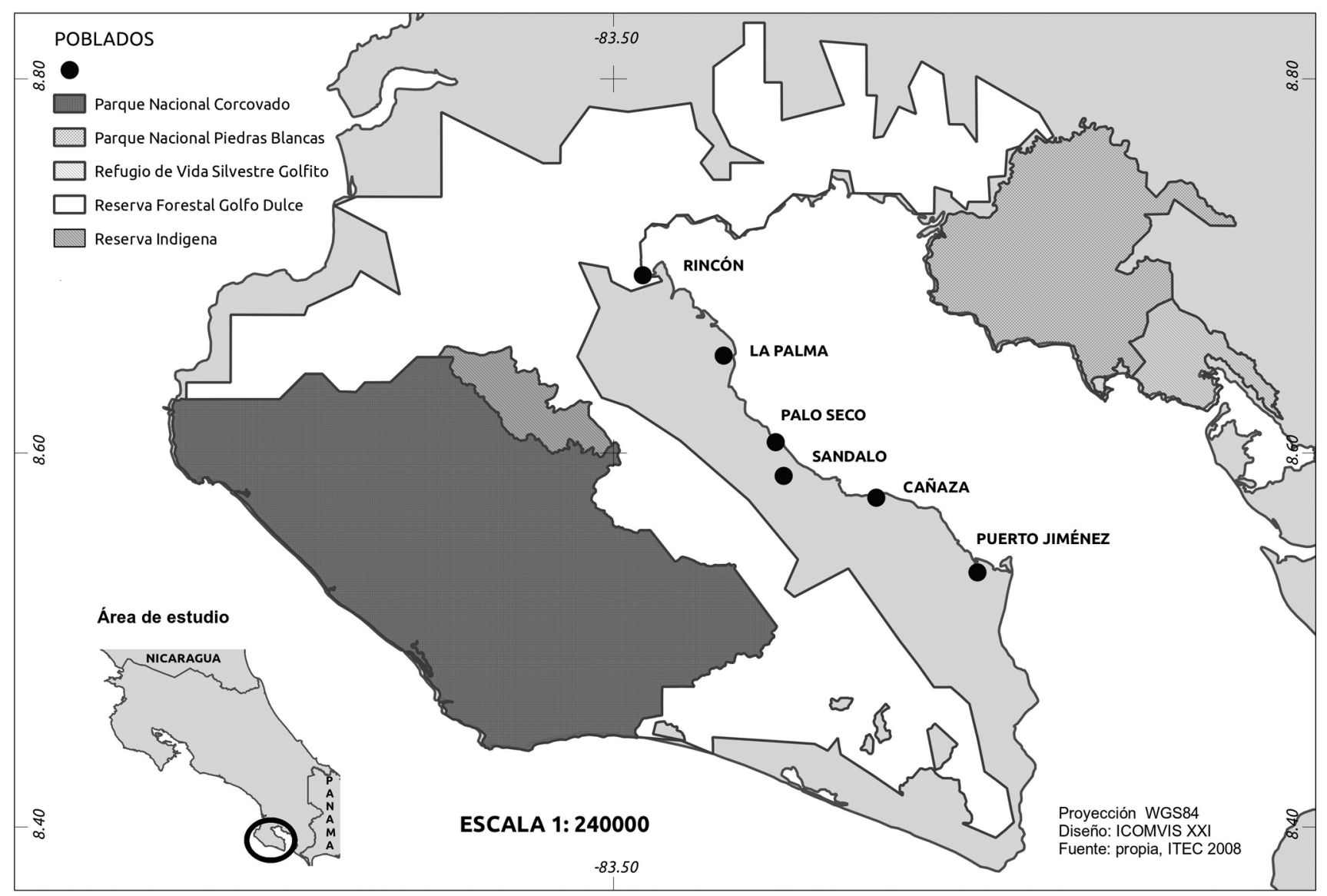

FIG. 1. Mapa del área de estudio detallando las áreas de conservación dentro de la Península de Osa, Costa Rica. Además, se incluyen las comunidades en donde se realizaron las entrevistas a los líderes religiosos durante el período de estudio.

discursos doctrinales. De esta manera, en primera instancia se elaboró una guía previa que exploró los siguientes tópicos de interés:

- Datos generales del líder religioso y de su iglesia.

- Visión de los libros sagrados sobre la naturaleza y los animales.

- Relación de su iglesia con la comunidad.

- Conocimiento personal del líder religioso hacia temas ambientales.

- Historia y cultura asociada a la cacería.

- Métodos utilizados para educar a sus miembros.

Las entrevistas fueron grabadas, siempre y cuando se contara con la autorización del entrevistado. También se tomaron apuntes donde se documentaba observaciones y comentarios sobre el tema. Las entrevistas duraron como máximo $2 \mathrm{~h}$ y se realizaron en grupos de 2 a 3 entrevistadores.

Para procesar las entrevistas en primer lugar se realizó la transcripción de las grabaciones. Luego se examinaron los temas emergentes y se los catalogó de acuerdo a un código de colores. Posteriormente se procedió a analizar cada tema emergente por separado, identificando citas claves para desarrollar conceptos y conclusiones de cada entrevista en particular y del tema en general.

\section{RESULTADOS}

Se realizaron 18 entrevistas, once en Puerto Jiménez, seis en La Palma y una en Cañaza. De los entrevistados 
once pertenecían a la Iglesia Evangélica (incluyendo un grupo de jóvenes), dos a la Iglesia Metodista, tres a la Iglesia Católica, una a la Iglesia de los Testigos de Jehová y, finalmente, una profesora de Moral y Religión en las escuelas de Puerto Jiménez y La Palma. A partir del análisis de las entrevistas, y para responder a los objetivos del trabajo, se identificaron tres categorías de análisis: a) percepción de los líderes con respecto a la naturaleza, b) percepción de los líderes con respecto a la cacería y c) métodos empleados por los líderes para educar a sus congregantes. A continuación se presenta la información que se obtuvo mediante las entrevistas a profundidad. Es necesario hacer notar que las palabras y frases transcritas literalmente de las entrevistas aparecen entre comillas.

\section{Percepción con respecto a la naturaleza}

Las respuestas dadas por los entrevistados arrojaron puntos de vista desiguales sobre la interpretación de los textos bíblicos; en todo caso los líderes coincidieron que el libro del Génesis es el que trata lo relativo a la naturaleza. Este libro tiene mucha importancia para ellos, puesto que fue citado por los líderes religiosos tanto cristianos como católicos.

Fue clara la referencia sobre la relación entre el ser humano y la naturaleza por parte de los miembros de las distintas congregaciones, sugiriendo que el cuidado de ésta es responsabilidad directa del hombre. Varios de los líderes evangélicos y un miembro de los Testigos de Jehová, coincidieron en que Dios Ilama al ser humano a ser "administrador" basándose en la Biblia argumentando que: "Dios creó primero los cielos, la tierra y los mares, así como las criaturas que allí habitan, y que posteriormente colocó al hombre en la Tierra con la responsabilidad principal cuidar de todo lo que fue creado por Dios". Hablaron también de la necesidad de utilizar los recursos de la Tierra como parte de la potestad de administrador que Dios concede al hombre, puntualizando la obligación del hombre de hacerlo racionalmente, sin sobreusos ni maltrato hacia los animales.

Una visión compartida por todos los líderes fue que hacer un mal uso de los recursos naturales es pecado y va en contra del mensaje de Dios. Algunos líderes religiosos evangélicos denominaron "pecado ambiental" a las acciones del hombre que atentan contra la naturaleza, mientras que una de las líderes católicas entrevistadas calificó de pecado "destruir la naturaleza y maltratar a los animales". Por su parte, una líder metodista enfatizó que "la mala organización de los recursos por parte del hombre es lo que está causando el deterioro ambiental, lo cual es incorrecto para Dios".
De acuerdo con las líderes católicas, Dios hace un Ilamado al hombre para que "someta" la naturaleza creada por él pero no a que la destruya. A la vez, estas religiosas comentaron: "la Iglesia llama a sus fieles a anunciar y denunciar injusticias que vayan en contra de la naturaleza, ya que la Iglesia Católica no contradice la teoría de la evolución $y$, como semejantes de Dios, debemos contribuir a proteger la obra de la creación".

Los líderes evangélicos respondieron al tema de la protección a la naturaleza mencionando que es muy difícil que el ser humano pueda hacer algo por cambiar tal situación, señalando que "la destrucción de la naturaleza es el proceso del pecado del hombre". Además, estos pastores añadieron que la destrucción de la naturaleza es producto de la falta de conocimiento del ser humano pero, a medida que el ser humano genera conocimiento, éste se ha ido contradiciendo, dañando la naturaleza en el proceso.

Los jóvenes miembros de una de las iglesias evangélicas afirmaron que los cristianos están llamados a "defender la naturaleza", a "poner el ejemplo", e incluso fueron más allá y aseguraron que deben "liderar el cuido del ambiente". Ellos añadieron que la naturaleza y los animales fueron dados por Dios a los hombres y "cumplen una función"; por esto y de acuerdo con ellos, la naturaleza debe ser conservada y "los animales no deben ser matados ni maltratados".

\section{Percepción con respecto a la cacería}

Según las entrevistas los tipos de cacería que se dan en la zona son por subsistencia, deporte y comercio. Ante esto, tanto líderes jóvenes como pastores, catequistas o maestras coincidieron en estar de acuerdo con el primer tipo de cacería ya que lo ven como un derecho que Dios da, pero no apoyaron las otras dos. Los líderes religiosos que hablaron sobre la cacería mencionaron que es una actividad tradicional y que hace parte del modo de vida de mucha gente, pero que ha ido disminuyendo con el paso de los años.

Ante esto, discutieron sobre las razones de esta práctica: "por necesidad de alimentarse: algunas personas de bajos recursos lo utilizan como un medio de subsistencia; por deporte; o para vender la carne y tener ingresos adicionales en los hogares". También señalaron que los locales cazan por recreación así como por la gran demanda de cazadores que no son de la zona que pagan a los campesinos para ir a las áreas de caza. En estos casos los entrevistados reconocieron que estos actos no son adecuados porque llevan implícito la matanza de otras especies que no son precisamente las codiciadas por los cazadores.

Dentro de las congregaciones evangélicas predominó el pensamiento de que el ser humano tiene una posición 
especial en el mundo y por tanto mayores responsabilidades para con él, incluyendo una caza responsable. Según sus argumentos, el hombre tiene una posición más importante que la de los animales en el sentido en que fue designado por Dios como "administrador de las cosas de Dios". Para poder cumplir este mandato divino, los líderes entrevistados consideraron que la gente puede cazar para comer y debería educarse acerca del tema. Sin embargo, ellos también comentaron que algunas personas piensan que de todas formas los animales se van a extinguir, por lo que no tendría mucho sentido protegerlos. De hecho, indicaron que hay enseñanzas bíblicas que expresan que el fin de los animales se va a dar, pero que de igual forma el hombre debe esforzarse por salvar lo más que se pueda. Algunos entrevistados afirmaron que un pensamiento común de los cazadores es que "Dios puso los animales ahíen el monte para usarlos y que nunca se iban a acabar porque nunca han escaseado".

Los líderes religiosos coincidieron en que el temor que existe entre los cazadores a ser reprendidos y sancionados por el MINAET ha promovido una disminución de la cacería en la zona. Los religiosos añadieron que "si alguna persona montea y es encontrada con carne puede ser llevada a la cárcel y decomisados los perros junto con los elementos de caza". En concordancia, mencionaron un cambio de mentalidad en algunos de los ex-cazadores que han sido contratados como guardaparques por parte del MINAET uniéndose a los esfuerzos para conservar la fauna local.

Los entrevistadores se refirieron poco a las posibles soluciones del tema de cacería. Sin embargo, muchos mencionaron la importancia de la educación, del aporte de materiales e incluso la posibilidad de implementar algunas medidas como zoocriaderos. No obstante, argumentaron que, en algunas ocasiones, el mismo MINAET representa un obstáculo por la cantidad de permisos que deben tenerse.

\section{Métodos empleados para educar a los congregantes}

En cuanto a la organización, la mayoría de las iglesias separa a sus miembros por grupos de edades para enseñar, a excepción de los Testigos de Jehová. Con los niños se utilizan técnicas variadas como teatro, payasos, mimos, danzas, leer cuentos, música, festivales y en algunos casos utilizan como apoyo libros educativos impresos por iglesias internacionales. Con los jóvenes y adultos se utilizan videos, charlas, talleres y también se han realizado campañas con actividades dirigidas a la comunidad.

Respecto a la disposición de los líderes para incorporar actividades relacionadas con la educación ambiental, tanto las catequistas de la Iglesia Católica como algunos pastores evangélicos y de la Iglesia Metodista se mostraron muy abiertos e interesados para escuchar propuestas de educación ambiental. Ante el cuestionamiento de proveer material relacionado con educación ambiental, algunos miembros de la fraternidad de pastores evangélicos resaltaron la importancia de examinar cuidadosamente los contenidos para que no contradigan a sus creencias o transmitan un mensaje con el que no están de acuerdo por completo.

Por otro lado, los Testigos de Jehová consideraron que es difícil trabajar en educación ambiental de forma local debido a que su estructura organizativa es muy estricta y siguen instrucciones dadas a nivel mundial. En consecuencia, queda poco margen para la incorporación de nuevas ideas y actividades propuestas por gente externa. A pesar de esto, los líderes de esta iglesia estuvieron abiertos ante la posibilidad de brindarles material informativo.

\section{DISCUSIÓN}

Las personas entrevistadas mostraron características en común a pesar de la diversidad de doctrinas religiosas; la principal de ellas es que su filosofía de vida gira en torno a las enseñanzas bíblicas. Esto también les ha provisto de una marcada sensibilidad hacia la naturaleza y los problemas ambientales actuales ya que ven reflejado el poder de Dios en la belleza escénica y en la compleja diversidad biológica. Por ejemplo, algunos pastores evangélicos señalaron que la Iglesia debe ser "Cristo-céntrica", es decir todo debe de girar en torno a Cristo y sus enseñanzas, dentro de las que se encuentra la naturaleza que es parte integral de Cristo y la creación de Dios. En consecuencia, el pensamiento de los líderes evangélicos indica que el cuidado y protección de los recursos naturales debe atribuirse al único hecho de ser la creación de Dios, pues ellos afirmaron que los hombres "tienen que tener cuidado de no darle tributo a la naturaleza, deben de darle tributo a Dios".

Los líderes religiosos entrevistados consideran al hombre como dueño de todo lo creado por Dios, lo cual está en su total disponibilidad. Por ejemplo, las líderes católicas hablaron sobre "someter", es decir, usar los recursos pero con medida, lo cual estaría más relacionado con el concepto de "administrar", utilizado por algunos líderes evangélicos. Sin embargo, el mal uso que se haga de estos recursos es visto de una forma negativa. En este sentido, llama la atención las respuestas dadas por los jóvenes miembros de una de las iglesias evangélicas. Acá fue posible observar cómo su percepción sobre la relación entre la doctrina religiosa y la protección de la naturaleza va más allá de la de los líderes adultos, ya que en ellos se evidenció una actitud de protección del medio ambiente mucho 
más activa y comprometida. Esto podría indicar que el mensaje religioso de protección de la naturaleza se está transmitiendo a las generaciones más jóvenes.

La población de la península de Osa se ha caracterizado por tener la cacería arraigada en su cultura, existiendo un patrón de consumo que sobrepasa la falta de control de esta actividad dentro de las áreas protegidas (Agenda XXI-ACOSA, 2004); por lo tanto, esta actividad no se ha desarrollado exclusivamente para subsistencia y complementación alimentaria sino también como una manera de fortalecer los vínculos familiares y sociales dentro de las comunidades. No obstante, este tema no fue tan mencionado por los entrevistados en comparación con otros problemas ambientales como la basura o la construcción de marinas, alegando que los últimos son más importantes. Incluso, varios entrevistados evitaron hablar sobre el tema de la cacería.

A pesar de esto, se evidenció una percepción de la cacería muy similar en los líderes que sí aceptaron hablar al respecto los cuales representaron todas las diferentes corrientes religiosas incluidas en este estudio. De este modo hubo una marcada generalización de aceptación sobre la caza de subsistencia por parte de los entrevistados cuando los casos lo ameriten, pero no así a la caza deportiva y mucho menos la caza para venta.

Si a esto se le suma el desarrollo eco-turístico que se ha llevado a cabo en la zona desde hace algunos años (Driscoll et al., 2011), se tiene que la población ha ido comprendiendo poco a poco el valor comercial que poseen los recursos aprovechados de otra manera que no sea la extracción, consumo o caza. De hecho las actividades comerciales de los pobladores han cambiado en torno a este fenómeno, tal es el caso de varios entrevistados que se dedican al turismo de naturaleza. Por lo tanto, ellos están conscientes del aporte económico que la biodiversidad y los recursos naturales les proporcionan si se conservan adecuadamente.

Lo anterior se podría traducir en un cambio de mentalidad acerca de la conservación y por consiguiente una buena disposición de aprender y conocer más a fondo tanto la problemática ambiental como las vías para su mitigación. Sin embargo, los líderes consideran fundamental la educación religiosa para la incorporación de conceptos relacionados con la ética, los valores y el estudio bíblico, siendo su prioridad educativa el desarrollo del componente espiritual, pasando los temas ambientales a un segundo plano. Por lo tanto, si bien están dispuestos a recibir educación ambiental, su prioridad es y seguirá siendo continuar con los compromisos de su fe.

Los programas y acciones a desarrollar en torno a la educación ambiental en la península de Osa, deben enfatizar los beneficios potenciales que podrían obtenerse a partir de los recursos naturales de maneras alternativas a los tradicionalmente utilizados. Además, es necesario proporcionar a los habitantes las bases teóricas necesarias para que logren comprender este complejo proceso y, fomentar así, que se formen su propio juicio acerca de la importancia de la conservación de los recursos naturales de la zona donde viven.

\section{Conclusiones}

En conclusión, se identificaron varios elementos dentro de las doctrinas religiosas que pueden ser empleados para canalizar estrategias de educación ambiental, como lo fueron el respeto a la naturaleza como creación de Dios, la transmisión de este mensaje hacia las generaciones jóvenes, la desaprobación a la cacería por deporte o comercio y la anuencia a incluir elementos de educación ambiental dentro del mensaje hacia las congregaciones. En este sentido, es importante que la información presentada en este trabajo sirva de base para ejecutar programas de educación ambiental, así como proyectos para la conservación de la biodiversidad de la península de Osa, que involucren a los líderes religiosos y a sus congregantes.

Los líderes religiosos comparten la visión de que el ser humano está por encima de la naturaleza y que ésta debe estar bajo el control de ellos. De acuerdo con ellos, el ser humano, como administrador de la naturaleza creada por Dios, está en la responsabilidad de cuidarla y protegerla, pero sólo por el hecho de ser creación de Dios.

Los representantes de las diferentes corrientes religiosas coinciden en que los recursos naturales están en total disponibilidad para el ser humano, pero éste debe hacer un uso racional de ellos.

El pensamiento de cuido y protección de lo creado por Dios se ve reflejado en los congregantes más jóvenes, los cuales enfatizan en la necesidad de una actitud más activa.

La cacería ilegal de fauna silvestre es un tema del que se habla poco dentro de las congregaciones religiosas, aun cuando exista aceptación de que la actividad continúa ejerciéndose y exista cierta tolerancia a la caza por subsistencia.

Los líderes religiosos de la península de Osa consideran que los principios éticos y morales son altamente prioritarios en la educación religiosa, lo cual dejaría a la educación ambiental dentro de las congregaciones en un plano secundario.

La inclusión de programas de educación ambiental dentro de las congregaciones religiosas en la península de Osa parece ser factible, siendo Los Testigos de Jehová la corriente religiosa más renuente ante esta propuesta. 


\section{AGRADECIMIENTOS}

Agradecemos a todos los entrevistados, miembros de las diferentes corrientes religiosas de Puerto Jiménez, La Palma y Cañaza por su disponibilidad a colaborar con este proyecto. Asimismo, agradecemos al Instituto Internacional en Conservación y Manejo de Vida Silvestre de la Universidad Nacional de Costa Rica por apoyar esta investigación.

\section{REFERENCIAS}

Agenda XXI-ACOSA. (2004). Agenda Ambiental: Área de Conservación Osa. Dirección de Sociedad Civil, MINAET: San José, Costa Rica.

Christen, C. (1994). Development and conservation on Costa Rica's Osa Peninsula, 1937-1977: A regional case study of historical land use policy and practice in a small neotropical country. Tesis de maestría. The Johns Hopkins University: Maryland, EEUU.

Dudley, N., Higgins-Zogib, L. \& Mansourian, S. (2005). Beyond Belief: Linking faiths and protected areas to support biodiversity conservation. WWF, Equilibrium and the Alliance of Religions and Conservation (ARC): Gland, Suiza.

Drews, C. (2003). The state of wild animals in the mind and households of a Neotropical society: The costarican case study. En Salem D. \& Rowan, A.N. (eds.). The States of the Animals II. D.C. Human Society Press: Washington D.C., E.E.U.U.

Driscoll, L., Hunt, C., Honey, M. \& Durham, W. (2011). The Importance of Ecotourism as a Development and Conservation Tool in the Osa Peninsula, Costa Rica. Center for Responsible Travel (CREST): Washington D.C., E.E.U.U.

ELAP. (2005). Caracterización general ecológica, social y económica del ACOSA. Proyecto Para la Elaboración de los Planes de Manejo de las Áreas Protegidas de ACOSA: San José, Costa Rica.

Fundación Marviva. (2009). Estrategia para el fortalecimiento y consolidación de alternativas productos y de organizaciones para la sostenibilidad económica y ambiental de los recursos marino-costeros en el Área de Conservación Osa. Proyecto Para la Elaboración de los Planes de Manejo de las Áreas Protegidas de ACOSA: San José, Costa Rica.

Herrera-MacBryde, O., Maldonado, T., Jiménez, V. \& Thömsen, K. (1997). Osa Península and Corcovado National Park, Costa Rica. En Davis, S.D., Heywood, V.H., Herrera-MacBryde, O., Villa-Lobos, J., \& Hamilton, A.(eds.). Centres of Plant Diversity: A Guide and Strategy for Their Conservation. Volume
3: The Americas. IUCN Publications Unit: Cambridge, Inglaterra.

Hernández, L., Wong, G., Mena, Y. \& Arguedas, S. (2008). Educación ambiental para la conservación de especies insignia en Osa. Ambientico 174.

Hernández, L., Wong, G., Mena, Y. \& Arguedas, S. (2011). Educación Ambiental como Estrategia para la Conservación de Fauna Silvestre en la Península de Osa, Costa Rica. Biocenosis $24(1-2)$

Kappelle, M., Castro, M., Acevedo, H., Gonzáles, L. \& Monge, H. (2002). Ecosistemas del Área de Conservación Osa. Editorial INBio: Heredia, Costa Rica.

Mansour, J. (1995). Parks in peril source book. The Nature Conservancy: America Verde: Virginia, E.E.U.U.

MIDEPLAN. (2003). Plan regional de desarrollo 2003-2006 región Brunca de Costa Rica. Recuperado de: http://www. mideplan.go.cr/component/option,com_docman/ task,doc_view/gid,33/

MINAE-SINAC. (2005). Estrategia Nacional para la Educación Ambiental en el SINAC 2005- 2010. Ministerio de Ambiente y Energía, Sistema Nacional de Áreas de Conservación: San José, Costa Rica.

Pérez, E. \& Ojasti, J. (1996). La utilización de la fauna silvestre en la América tropical y recomendaciones para su manejo sustentable en las sabanas. Ecotrópicos 9.

Rosero-Bixby, L., Maldonado-Ulloa, T. \& Bonilla-Carrión, R. (2002). Bosque y población en la Península de Osa, Costa Rica. Revista Biología Tropical 50, 585-598.

Tosi, J.A. (1969). Mapa ecológico según la clasificación de Zonas de Vida del Mundo de L.R. Holdridge. Centro Científico Tropical: San José, Costa Rica.

UNESCO. (1977). Tendencias de la Educación Ambiental. UNESCO: Tbilisi, URSS.

Valles, M.S. (1997). Técnicas cualitativas de investigación social. Reflexión metodológica y práctica profesional. Síntesis Sociología: Madrid, España

Vargas-Mena, E. (2006). Educación ambiental para la conservación de especies amenazadas en la Península de Osa, Costa Rica (2004-2005). Informe de sistematización de proyecto MINAE, SINAC, UNED, CEA, UNA, ICOMVIS, EDECA: Costa Rica.

Wong, G. (2006). Un Tesoro Amenazado: Osa y su Fauna Silvestre. Mimeógrafo. Impreso en el Proyecto: Educación Ambiental para la Conservación de Especies Insignia de la Península de Osa, Costa Rica. SINAC-UNED-UNA: Costa Rica. 
